\title{
A THEOREM CONCERNING THE GEODESICS ON A PARABOLOID OF REVOLUTION ${ }^{1}$
}

\author{
DONALD LING AND LEON RECHT
}

We shall examine the behavior in the large of the geodesics on a paraboloid of revolution. It will appear that on every geodesic there is a unique point - the "point of symmetry"-which divides the geodesic into two "conjugate rays," which are mirror images of each other in the plane determined by that point and the axis of the paraboloid. Every geodesic except the meridians, which have no singularities whatever, has infinitely many double points, but no other singular points. If, starting at the point of symmetry, we imagine the conjugate rays of a geodesic to be traversed simultaneously by two points which are images in the plane of symmetry, and which continue until they meet for the first time-at the "first double point" of the geodesic-we shall have generated a "loop of type 1." If, starting at the point of symmetry, they pass through the first double point and continue until they next meet - at the "second double point"-we shall have formed a "loop of type 2." We define in this way loops of types $1,2,3, \cdots$. The last double point to be reached in the process of generating a loop of a given type will be called the "vertex" of the loop.

The main result of this paper is the following: The paraboloid can be divided by planes perpendicular to the axis into infinitely many zones, each zone to include the points of that bounding circle which is nearer the vertex. The zone containing the vertex we call the "cap," the following the "first zone," and so on. The zones have the following properties:

1. Each interior point of the $k$ th zone is the vertex of exactly two distinct loops of each of the types $1,2, \cdots, k$, but is the vertex of no loop of type greater than $k$.

2. Each boundary point in the $k$ th zone is the vertex of two loops of each of the types $1,2, \cdots, k-1$ and of one loop of type $k$. It is the vertex of no loop of type greater than $k$.

3. No point of the cap is the vertex of any loop.

Let the paraboloid be that obtained by revolving $r^{2}=x$ about the $x$ axis. As coordinates of a point $P$ on the surface (the vertex excepted) we take $r$ to be the length of the vector $R$ from $P$ perpendicular to the axis and directed toward $P$, and $\theta$ to be the angle which the plane determined by $P$ and the axis makes with an arbitrary plane contain-

\footnotetext{
${ }^{1}$ Presented to the Society, February 24, 1940.
} 
ing the axis. The differential equation defining the geodesics (cf. Darboux, Leçons sur la Théorie Générale des Surfaces..., vol. 3) is

$$
\frac{d \theta}{d r}=-\frac{c}{r}\left[\frac{4 r^{2}+1}{r^{2}-c^{2}}\right]^{1 / 2}
$$

where $c$ is an arbitrary constant. Corresponding to $c=0$ are the meridians $\theta=$ const. Since their nature is obvious we may except them from consideration, and assume $c \neq 0$. The solution of (1) corresponding to a given value of $c \neq 0$ and passing through $\left(r_{0}, \theta_{0}\right)$ is

$$
\begin{aligned}
\theta= & \theta_{0}+\arctan c\left[\frac{4 r^{2}+1}{r^{2}-c^{2}}\right]^{1 / 2}-c \log \frac{\left(4 r^{2}+1\right)^{1 / 2}+2\left(r^{2}-c^{2}\right)^{1 / 2}}{\left(4 r^{2}+1\right)^{1 / 2}-2\left(r^{2}-c^{2}\right)^{1 / 2}} \\
& \quad-\arctan c\left[\frac{4 r_{0}^{2}+1}{r_{0}^{2}-c^{2}}\right]^{1 / 2}+c \log \frac{\left(4 r_{0}^{2}+1\right)^{1 / 2}+2\left(r_{0}^{2}-c^{2}\right)^{1 / 2}}{\left(4 r_{0}^{2}+1\right)^{1 / 2}-2\left(r_{0}^{2}-c^{2}\right)^{1 / 2}} \\
= & \theta_{0}+f\left(r ; r_{0}, c\right), \text { say. }
\end{aligned}
$$

As $r \rightarrow|c|$ the right member of (2) approaches

$$
\begin{aligned}
\bar{\theta}=\theta_{0} & +\frac{\pi}{2} \operatorname{sign} c-\arctan c\left[\frac{4 r_{0}^{2}+1}{r_{0}^{2}-c^{2}}\right]^{1 / 2} \\
& +c \log \frac{\left(4 r_{0}^{2}+1\right)^{1 / 2}+2\left(r_{0}^{2}-c^{2}\right)^{1 / 2}}{\left(4 r_{0}^{2}+1\right)^{1 / 2}-2\left(r_{0}^{2}-c^{2}\right)^{1 / 2}} .
\end{aligned}
$$

It can be shown without difficulty that $(|c|, \bar{\theta})$ is a point of the corresponding geodesic. ${ }^{2}$ It is apparent from (2) and (3) that if $c<0 \theta$ increases monotonically from $\bar{\theta}$ at $r=|c|$ to $\infty$ at $r=\infty$, while if $c>0$ $\theta$ decreases monotonically from $\bar{\theta}$ at $r=|c|$ to $-\infty$ at $r=\infty$.

Consider the two solutions

$$
\begin{aligned}
\theta^{(1)} & =\theta_{0}+f\left(r ; r_{0}, c\right), \\
\theta^{(2)} & =\left\{\theta_{0}+2 f\left(|c| ; r_{0}, c\right)\right\}+f\left(r ; r_{0},-c\right) \\
& =\left\{\theta_{0}+2 f\left(|c| ; r_{0}, c\right)\right\}-f\left(r ; r_{0}, c\right) .
\end{aligned}
$$

It is clear that $\theta^{(1)}(|c|)=\theta^{(2)}(|c|)=\bar{\theta}$. Moreover, at the point $(|c|, \bar{\theta})$ the two solutions have a common tangent, and thus belong to the same geodesic. Furthermore

$$
\theta^{(1)}(r)-\bar{\theta}=f\left(r ; r_{0}, c\right)-f\left(|c| ; r_{0}, c\right)=-\left[\theta^{(2)}(r)-\bar{\theta}\right]
$$

${ }^{2}$ It is readily verified that if $r$ and $\theta$ are expressed in terms of the arc length $s$ along the geodesic measured from $\left(r_{0}, \theta_{0}\right)$, then $r \rightarrow|c|$ and $\theta \rightarrow \vec{\theta}$ as $s$ approaches a certain finite limit. 
so that the points $\left(r, \theta^{(1)}(r)\right)$ and $\left(r, \theta^{(2)}(r)\right)$ are mirror images of each other in the plane determined by the axis and the point $(|c|, \bar{\theta})$. This is the point of symmetry of the geodesic, and equations (4) give a pair of conjugate rays as defined above. Bearing in mind that $\theta^{(1)}(r)$ and $\theta^{(2)}(r)$ are monotonic in opposite senses, and that the absolute value of each is large with $r$, we see that each geodesic has infinitely many double points, but no other multiple points.

Assume $\theta_{0}=0$. Then $\bar{\theta}$ is the angle through which the radius vector $R$ turns as $(r, \theta)$ moves along a geodesic ray from its initial point $\left(r_{0}, 0\right)$ to its point of symmetry $(|c|, \bar{\theta})$. We shall study the variation of $\bar{\theta}$ as the parameter $c$, which controls the direction of the geodesic ray at its initial point $\left(r_{0}, 0\right)$, varies over non-negative values.

We find from (3) for $c>0$ that

$$
\frac{d \bar{\theta}}{d c}=-\lambda+\log \frac{\lambda+2}{\lambda-2} ; \quad \lambda=\left[\frac{4 r_{0}^{2}+1}{r_{0}^{2}-c^{2}}\right]^{1 / 2} .
$$

As $\lambda$ increases from 2 to $\infty$ the logarithm in (5) decreases monotonically from $\infty$ to 0 . Thus $d \bar{\theta} / d c=0$ has a single root $\lambda_{0}>2$. In terms of $c$, from the second of equations (5), $d \bar{\theta} / d c$ vanishes when and only when $c=c^{*}=\left(\left(\lambda_{0}^{2}-4\right) r_{0}^{2}-1\right)^{1 / 2}$. This root is real if, as we now assume, $r_{0} \geqq 1 /\left(\lambda_{0}^{2}-4\right)^{1 / 2}$. If $c>c^{*}, d \bar{\theta} / d c<0$; if $c<c^{*}, d \bar{\theta} / d c>0$. Since $\bar{\theta}\left(r_{0}, c\right)$ is continuous in $c$ for $0 \leqq c<r_{0}$, and since $\bar{\theta}\left(r_{0}, 0\right)=\pi / 2$ and $\lim _{c \rightarrow r_{0}} \bar{\theta}\left(r_{0}, c\right)=0, \bar{\theta}\left(r_{0}, c\right)$ attains its greatest value, $\theta^{*}\left(r_{0}\right)$, at $c=c^{*}$.

From (3) and (5)

$$
\begin{aligned}
\theta^{*}\left(r_{0}\right)= & \pi / 2-\arctan \lambda_{0}\left(\left(\lambda_{0}^{2}-4\right) r_{0}^{2}-1\right)^{1 / 2} \\
& +\lambda_{0}\left(\left(\lambda_{0}^{2}-4\right) r_{0}^{2}-1\right)^{1 / 2}
\end{aligned}
$$

This function is continuous for $r_{0} \geqq 1 /\left(\lambda_{0}^{2}-4\right)^{1 / 2}$ and its derivative, $\left(\left(\lambda_{0}^{2}-4\right) r_{0}^{2}-1\right)^{1 / 2} / r_{0}$, is positive for $r_{0} \geqq 1 /\left(\lambda_{0}^{2}-4\right)^{1 / 2}$. Thus $\theta^{*}\left(r_{0}\right)$, which gives $\bar{\theta}$ for the geodesic ray of greatest $\bar{\theta}$ through $\left(r_{0}, 0\right)$, is continuous and strictly increasing. Moreover $\theta^{*}\left(1 /\left(\lambda_{0}^{2}-4\right)^{1 / 2}\right)=\pi / 2$ and $\lim _{r_{0} \rightarrow \infty} \theta^{*}\left(r_{0}\right)=\infty$. There is, then, a sequence of values of $r_{0}: r_{0}^{(1)}<r_{0}^{(2)}<r_{0}^{(3)}<\cdots$ such that $\theta^{*}\left(r_{0}^{(k)}\right)=k \pi(k=1,2, \cdots)$. The portion of the paraboloid defined by $r_{0}^{(k)} \leqq r_{0}<r_{0}^{(k+1)}$ we shall call the " $k$ th zone," and the parallel $r_{0}=r_{0}^{(k)}$ its boundary. Let $r_{0}$ be an interior point of the $k$ th zone; then $k \pi<\theta^{*}\left(r_{0}\right)<(k+1) \pi$. As $c$ increases from 0 to $c^{*}, \bar{\theta}\left(r_{0}, c\right)$ increases from $\pi / 2$ to $\theta^{*}\left(r_{0}\right)$, and as $c$ then increases from $c^{*}$ to $r_{0} \theta\left(r_{0}, c\right)$ decreases from $\theta^{*}\left(r_{0}\right)$ to 0 . Hence there are two sequences of values of $c,\left\{c_{1}^{(1)}, \cdots, c_{k}^{(1)}\right\}$ and $\left\{c_{1}^{(2)}, \cdots, c_{k}^{(2)}\right\}$ satisfying $c_{1}^{(1)}<c_{2}^{(1)}<\ldots<c_{k}^{(1)}<c^{*}<c_{k}^{(2)}<\cdots<c_{1}^{(2)}$ and having the property that $\bar{\theta}\left(r_{0}, c_{m}^{(1)}\right)=\bar{\theta}\left(r_{0}, c_{m}^{(2)}\right)=m \pi,(m=1, \cdots, k)$. When $r_{0}$ is 
on the boundary of the $k$ th zone, $c_{k}^{(1)}=c^{*}=c_{k}^{(2)}$; but, for $m<k, c_{m}^{(1)}$ and $c_{m}^{(2)}$ are distinct.

Interpreted geometrically this means that through a point $\left(r_{0}, 0\right)$ in the $k$ th zone there are, for each value of $m=1, \cdots, k$, two geodesic rays for which $\bar{\theta}=m \pi$. The conjugate ray of each, being a reflection of the original ray, clearly passes through $\left(r_{0}, 2 m \pi\right)$, which is identical with $\left(r_{0}, 0\right)$. This point is therefore the vertex of two loops of each of the types $1, \cdots, k$. If $\left(r_{0}, 0\right)$ is on the boundary of the $k$ th zone this statement must be modified to the effect that the loops of type $k$ are coincident, those of lower types remaining distinct.

It remains to examine the cap, that is, that portion of the surface for which $r_{0}<r_{0}^{(1)}$. If $1 /\left(\lambda_{0}^{2}-4\right)^{1 / 2} \leqq r_{0}<r_{0}^{(1)}, \theta^{*}\left(r_{0}\right)<\pi$ and no loop with vertex $\left(r_{0}, 0\right)$ is possible. If $r_{0}<1 /\left(\lambda_{0}^{2}-4\right)^{1 / 2}$, then $\lambda=\left[\left(4 r_{0}^{2}+1\right) /\left(r_{0}^{2}-c^{2}\right)\right]^{1 / 2} \geqq\left(4+1 / r_{0}^{2}\right)^{1 / 2}>\lambda_{0}$ and $d \bar{\theta} / d c<0$. As $c$ increases from 0 to $r_{0}, \bar{\theta}\left(r_{0}, c\right)$ decreases from $\pi / 2$ to 0 . Thus no loop having a vertex in this portion is possible. We have now established all the results stated in the introduction.

The numbers $r_{0}^{(n)}$ can be calculated from (6). For, if we set $\theta^{*}\left(r_{0}\right)=n \pi$ and replace $\left(\left(\lambda_{0}^{2}-4\right) r_{0}^{2}-1\right)^{1 / 2}$ by $\mu, \quad(6)$ becomes $\mu-\operatorname{arc} \tan \mu=(2 k-1) \pi / 2$. Since the left member increases monotonically from 0 to $\infty$ as $\mu$ increases from 0 to $\infty$, there is exactly one root $\mu_{n}$ for each value of $r=1,2,3, \cdots$, and $r_{0}^{(n)}=\left[\left(\mu_{n}^{2}+1\right) /\left(\lambda_{0}^{2}-4\right)\right]^{1 / 2}$. For large values of $n, \mu_{n}$ is of the order of $n \pi$, and $r_{0}^{(n)}$ is of the order of $\left[\left(n^{2} \pi^{2}+1\right) /\left(\lambda_{0}^{2}-4\right)\right]^{1 / 2}$. We have approximately $\lambda_{0}=2.40$ and $1 /\left(\lambda_{0}^{2}-4\right)^{1 / 2}=3 / 4$. Thus $3 n \pi / 4$ furnishes an estimate of $r_{2}^{(n)}$ for large values of $n$.

Columbia University 